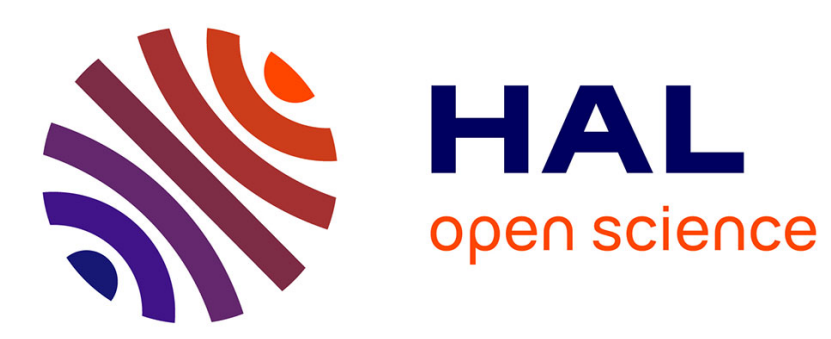

\title{
Rational countable steps functions on the Circle and ergodicity of Maharam measures
}

\author{
Julien Brémont
}

\section{To cite this version:}

Julien Brémont. Rational countable steps functions on the Circle and ergodicity of Maharam measures. Bulletin of the London Math. Soc., 2013, 1 (1), pp.1-13. hal-00794138

\section{HAL Id: hal-00794138 \\ https://hal.science/hal-00794138}

Submitted on 25 Feb 2013

HAL is a multi-disciplinary open access archive for the deposit and dissemination of scientific research documents, whether they are published or not. The documents may come from teaching and research institutions in France or abroad, or from public or private research centers.
L'archive ouverte pluridisciplinaire HAL, est destinée au dépôt et à la diffusion de documents scientifiques de niveau recherche, publiés ou non, émanant des établissements d'enseignement et de recherche français ou étrangers, des laboratoires publics ou privés. 


\title{
Rational countable steps functions on the Circle and ergodicity of Maharam measures
}

\author{
Julien Brémont
}

\begin{abstract}
We consider skew-products defined by BV countable steps functions with rational endpoints over an irrational rotation on the circle. We study the ergodicity of Lebesgue measure and more generally of all conformal (also called Maharam) measures. We next give an application to BV Davenport series.
\end{abstract}

\section{Introduction}

Let $\mathbb{T}=\mathbb{R} / \mathbb{Z}$ be the circle endowed with an irrational rotation $T x=x+\alpha \bmod 1$ and Lebesgue measure $\mu$. Let $f: \mathbb{T} \rightarrow \mathbb{R}$ be measurable, bounded and with $\mu$-zero mean, i.e. $\int f d \mu=0$. We set $T f=f \circ T$ and introduce the ergodic sums :

$$
f_{n}(x)=\sum_{k=0}^{n-1} T^{k} f(x), x \in \mathbb{T}, n \geq 1 .
$$

A usual way of studying these sums is via the skew-product associated with $f$ :

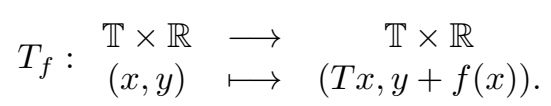

The asymptotic behaviour of $\left(f_{n}(x)\right)$ for a $\mu$-typical $x$ is related to the ergodic properties of the uniform measure $\nu=\mu \otimes \lambda_{\mathbb{R}}$ on $\mathbb{T} \times \mathbb{R}$, which is naturally $T_{f}$-invariant. It is well-known that this question of ergodicity is directly connected with the coboundary equation $f=g-T g, \mu$-a.-e., and more generally with equations $f-g+T g \in H, \mu$-a.-e., for a closed subgroup $H$ of $(\mathbb{R},+)$; cf for example Schmidt [20]. Let us recall that when $f$ is not a measurable coboundary, then for $\mu$-a.-e. $x \in \mathbb{T}$ the proportion of $n \leq N$ so that $f_{n}(x)$ belongs to a fixed bounded domain tends to zero as $N \rightarrow+\infty$. Ergodicity furthermore implies that $\left(f_{n}(x)\right)_{n \geq 1}$ is dense in $\mathbb{R}$ for $\mu$-a.-e. $x \in \mathbb{T}$ and gives for this sequence statistics described by $\nu$.

An important question is to determine all Borel locally finite (Radon) $T_{f}$-invariant ergodic measures on $\mathbb{T} \times \mathbb{R}$, for example in order to find points with different behaviours for the ergodic sums. In this direction, one commonly introduces a family of $T_{f}$-invariant Radon measures associated with $f$. For each $t \in \mathbb{R}$ define the Maharam measure $\nu_{t, f}$ on $\mathbb{T} \times \mathbb{R}$ by :

$$
\int_{\mathbb{T} \times \mathbb{R}} g(x, y) d \nu_{t, f}(x, y)=\int_{\mathbb{T} \times \mathbb{R}} g(x, y) e^{t y} d \mu_{t, f}(x) d y,
$$

where $\mu_{t, f}$ is a Borel probability measure on $\mathbb{T}$ verifying the quasi-invariance property :

$$
d T \mu_{t, f}=e^{t T^{-1} f} d \mu_{t, f}
$$

The measure $\mu_{t, f}$ exists and is uniquely defined as well as non-atomic as soon as $f$ is BV, which will be our situation. These facts are for instance detailed in Conze-Guivarc'h [8]. All measures $\nu_{t, f}$ are $T_{f}$-invariant, with $\nu_{0, f}=\nu$, and a natural problem is whether they are ergodic and then the only $T_{f}$-invariant ergodic Radon measures on $\mathbb{T} \times \mathbb{R}$. 
Let $\mathbb{Q}(\mathbb{T})$ denote the set of rationals in $\mathbb{T}$, often seen as $\mathbb{Q} \cap[0,1)$. Set $\mathbb{Q} *(\mathbb{T})=\mathbb{Q}(\mathbb{T}) \backslash\{0\}$. Our purpose in this article is to study the ergodicity of Maharam measures for the following examples :

$$
U(x)=\sum_{r \in \mathbb{Q}^{*}(\mathbb{T})} u_{r}\left(1_{[0, r)}(x)-r\right), \text { where } u=\left\{u_{r}\right\}_{r \in \mathbb{Q}^{*}(\mathbb{T})} \in l^{1} .
$$

Extensions with maps of this form naturally appear for example when analyzing the ergodic properties of billiard flows through the introduction of Poincaré sections. Let $U\left(x^{-}\right)$and $U\left(x^{+}\right)$be the left and right limits of $U$ at $x$. Notice first that $U$ can be uniquely written in the form (1.2), because $U\left(r^{-}\right)-U\left(r^{+}\right)=u_{r}$ for all $r \in \mathbb{Q}^{*}(\mathbb{T})$. Introduce :

$$
\rho_{u}=\sum_{r \in \mathbb{Q}^{*}(\mathbb{T})} r u_{r}
$$

We have $U\left(0^{+}\right)=-\rho_{u}$. Let now $H_{u}$ be the closed subgroup of $(\mathbb{R},+)$ generated by the values taken by $U$. Denoting by $\sigma(A)$ the subgroup generated by a set $A$, the previous remarks show that:

$$
H_{u}=\overline{\sigma\left(u \cup\left\{\rho_{u}\right\}\right)} .
$$

Observe that $H_{u}=\mathbb{R}$ if and only if $\overline{\sigma(u)}=\mathbb{R}$. In the sequel we write $T_{u}$ for $T_{U}$ and $\mu_{t, u}$ for $\mu_{t, U}$. Let $\lambda_{H}$ be Haar measure on a closed subgroup $H$ of $(\mathbb{R},+)$. The story taking place in $\mathbb{T} \times H_{u}$, introduce the family of Radon $T_{u}$-invariant measures $\nu_{t, u}$ on $\mathbb{T} \times H_{u}, t \in \mathbb{R}$, defined by :

$$
d \nu_{t, u}(x, y)=e^{t y} d \mu_{t, u}(x) d \lambda_{H_{u}}(y) .
$$

For simplicity we say that $T_{u}$ is $t$-ergodic on $\mathbb{T} \times H_{u}$ if $\left(\mathbb{T} \times H_{u}, \mathcal{B}\left(\mathbb{T} \times H_{u}\right), T_{u}, \nu_{t, u}\right)$ is ergodic, that $U$ is a $t$-coboundary if $U=g-T g, \mu_{t, u}$-a.-e., for a real measurable $g$ (which can always be chosen $H_{u}$-valued). Conveniently we use ergodic for 0-ergodic and coboundary for 0-coboundary.

The problem of the ergodicity of Lebesgue measure for compact extensions by finite step functions has been intensively studied; cf Veech [21] for instance. The case of non-compact extensions by finite step functions and the ergodicity of Lebesgue measure has also been considered for a long time. This question is sensibly more difficult, because of the possible phenomenon of non-regularity (recalled below). We adress the reader to the recent paper by Conze [7] and references therein. In [7] several existing results are extended. The case of Maharam measures is also treated in particular situations, improving former results of Aaronson-Nakada-Sarig-Solomyak [2]. The analysis focuses on the properties of the sequence $\left(q_{n} \rho_{u}\right)_{n}$, where $\left(q_{n}\right)$ are the denominators of the convergents of $\alpha$. We give here partial results complementary to those in [7]. Our method is sensibly different, working even if $\rho_{u}=0$, but with main focus the case of rational endpoints and $u \in l^{1}$.

Mention an interesting technical point making the presentation easier. Introduce the sawtooth function $\{x\}^{\star}=x-\lfloor x\rfloor-1 / 2$, where $\lfloor x\rfloor$ is the integer part of $x$, with $\{0\}^{\star}=0$. We have :

$$
\{x\}^{\star}=-\sum_{m \geq 1} \frac{\sin (2 \pi m x)}{\pi m}, x \in \mathbb{T} .
$$

It is easy to check that $1_{[0, r)}(x)-r=\{x-r\}^{\star}-\{x\}^{\star}$. Defining $v=\left\{v_{r}\right\}_{r \in \mathbb{Q}(\mathbb{T})}$ by $v_{r}=u_{r}, r \neq 0$, and $v_{0}=-\sum_{r \in \mathbb{Q}^{*}(\mathbb{T})} u_{r}$, one can rewrite $(1.2)$ as :

$$
U(x)=\sum_{r \in \mathbb{Q}(\mathbb{T})} v_{r}\{x-r\}^{\star} .
$$

We will use both representations for $U$. In practice $\{x\}^{\star}$ has only one discontinuity on $\mathbb{T}$, whereas $1_{[0, r)}-r$ has two (when $0<r<1$ ). It also behaves very well under rational rotations, as illustrated by the following identities, checked for instance on the Fourier series :

$$
\forall q \geq 1, \sum_{0 \leq k<q}\{x+k / q\}^{\star}=\{q x\}^{\star}
$$




\section{Notations}

- The convergents of $\alpha$ are denoted by $\left(p_{n} / q_{n}\right)$ and its partial quotients by $\left(a_{n}\right)$. We will use classical inequalities, which can for instance be found in Khinchin [15]. Introduce also :

* (C) : $\left(a_{n}\right)$ is bounded or $\forall A>0 \exists B \geq A \exists(\psi(n)) \rightarrow+\infty$ so that $A \leq a_{\psi(n)} \leq B$.

* $\left(C^{\prime}\right): \forall A>0 \exists B \geq A \exists(\psi(n)) \rightarrow+\infty$ with $A \leq a_{\psi(n)+1} \leq B \& \min _{p>1}, p \mid q_{\psi(n)}\{p\} \rightarrow+\infty$.

- A rational $r=p / q \in \mathbb{Q}(\mathbb{T})$ is implicitly written in irreducible form with $q \geq 1$. We set $d(r)=q$. Let $\mathcal{P}$ be the set of primes. The ged of integers $n$ and $m$ is $(n, m)$. Denote by $\varphi$ Euler's Totient function, where $\varphi(n)$ is the number of integers less than or equal to $n$ and prime with $n$. Recall the classical identity $\sum_{q \mid n} \varphi(q)=n, n \geq 1$.

- The variation of a function $f$ is written as $V(f)$. If $f$ is BV, the sum of its jumps is $J(f)$. The image of $\mu_{t, f}$ by $f$ is written as $\mathcal{L}_{t}(f)$, with $\mathcal{L}_{0}(f)=\mathcal{L}(f)$.

- The distance in $\mathbb{T}$ or $\mathbb{R}$ is denoted by $d(x, y)$, the distance from a real $x$ to $\mathbb{Z}$ by $((x))$.

\section{Essential values}

Let $H$ be a closed subgroup of $(\mathbb{R},+)$ and $f: \mathbb{T} \rightarrow H$ be measurable. As $T_{f}$ is a cylindrical transformation of $\mathbb{T} \times H$ with an ergodic basis, the question of the ergodicity of $T_{f}$ can be reduced to the study of the set of periods or "essential values". Basic results for $\mu$ were developed by Schmidt [20] and Feldman-Moore [13]. The same ones for $\mu_{t, f}, t \in \mathbb{R}$, can be found in ConzeRaugi $[\mathbf{9}]$, section 2.3. Recall that the notation $f_{n}$ is extended as usual to $n \in \mathbb{Z}$ by setting $f_{-n}(x)=$ $-T^{-n} f_{n}(x), n \geq 0$. Denote also by $\bar{H}=H \cup\{\infty\}$ the one-point compactification of $H$.

Definition 3.1. An element $c \in \bar{H}$ is a $t$-essential value for $f$ if for any neighbourhood $V$ of $c$ and $A \in \mathcal{B}(\mathbb{T})$ with $\mu_{t, f}(A)>0$ there is $n \in \mathbb{Z}$ so that $\mu_{t, f}\left(A \cap T^{-n} A \cap\left\{f_{n} \in V\right\}\right)>0$.

The set of $t$-essential values is written as $\overline{\mathcal{E}_{t}(f)} \subset \bar{H}$. The finite part $\mathcal{E}_{t}(f)$ is a closed subgroup of $(H,+)$. Let us set $\mathcal{E}_{0}(f)=\mathcal{E}(f)$. An important property, called $t$-regularity, is when $f-g+T g \in$ $\mathcal{E}_{t}(f), \mu_{t, f}$-a.-e., for a real measurable $g$. We say regular for 0 -regular. For instance, a $t$-measurable coboundary or a $t$-ergodic cocycle on $\mathbb{T} \times H$ are $t$-regular. Mention that a cocycle may not be regular (cf Zimmer [22], when $t=0$ ). In our context, we have (see $[\mathbf{2 0 , 9}]$ ) :

\section{Proposition 3.2.}

i) The cocycle defined by $f$ is t-ergodic on $\mathbb{T} \times H$ if and only if $\mathcal{E}_{t}(f)=H$.

ii) The function $f$ is a $t$-coboundary if and only if $\overline{\mathcal{E}_{t}(f)}=\{0\}$.

iii) The cocycle defined by $f$ is $t$-non-regular if and only if $\overline{\mathcal{E}_{t}(f)}=\{0, \infty\}$.

A way of finding finite essential values is via the following lemma; this is an extension of proposition 9 in Lemańczyk-Parreau-Volny [17] to the conformal case. We suppose that $f$ is BV.

LEMMA 3.3 .

Let $\left(k_{n}\right) \rightarrow+\infty$, with $\left(k_{n}\right)$ extracted from $\left(q_{n}\right)$ when $t \neq 0$. If $k_{n} \alpha \rightarrow 0$ mod 1 and $\mathcal{L}_{t}\left(f_{k_{n}}\right)$ converges to a measure $m$ for the vague topology, then $\operatorname{supp}(m) \subset \mathcal{E}_{t}(f)$.

Proof of the lemma : One way is to follow the proof of lemma 2.2 in $[\mathbf{3}]$ given for $t=0$. The arguments are the same, except for the first step proving that $\int_{\mathbb{T}}\left|g-T^{k_{n}} g\right| d \mu_{t, f} \rightarrow 0$, as $n \rightarrow$ $+\infty$, for a bounded and measurable $g$. This is clearly true for a continuous $g$. For a general $g$, let $\varepsilon>0$ and take a continuous $h$ so that $\int_{\mathbb{T}}|g-h| d \mu_{t, f}<\varepsilon$. It is then enough to show that $\int_{\mathbb{T}}\left|T^{k_{n}} g-T^{k_{n}} h\right| d \mu_{t, f}$ is small. From the quasi-invariance property, the latter equals :

$$
\int_{\mathbb{T}}|g-h| e^{-t f_{k_{n}}} d \mu_{t, f} \leq e^{|t| V(f)} \int_{\mathbb{T}}|g-h| d \mu_{t, f} \leq e^{|t| V(f)} \varepsilon,
$$


where we have used the Denjoy-Koksma inequality. This completes the proof of the lemma.

\section{Results}

Lemma 4.1. Conditions $(C)$ and $\left(C^{\prime}\right)$ are both true $\mu$-a.e.e.

Our purpose is to establish :

Theorem 4.2. Let $\alpha \notin \mathbb{Q}$ and $u=\left\{u_{r}\right\}_{r \in \mathbb{Q}^{*}(\mathbb{T})} \in l^{1}$.

i) - If $\operatorname{supp}(u)$ is finite or $(C)$ is true then $U$ is regular.

- If $U$ is regular, then $T_{u}$ is ergodic in $\mathbb{T} \times H_{u}$.

ii) If $\left(C^{\prime}\right)$ is true, then $T_{u}$ is $t$-ergodic on $\mathbb{T} \times H_{u}$, for all $t \in \mathbb{R}$.

Remark. - As a corollary $U$ is a coboundary if and only if $u=0$. For $\mu$-a.-e. angle $\alpha$, this is also true for $t$-coboundaries, for all $t \in \mathbb{R}$.

Remark. - The first item of the theorem is an improvement of a result in $[\mathbf{6}]$, where ergodicity in $\mathbb{T} \times(1 / d(r)) \mathbb{Z}$ is shown for $U(x)=1_{[0, r)}(x)-r, r \in \mathbb{Q}^{*}(\mathbb{T})$. One can build other kinds of examples. For instance for any rational $0<\beta<1 / 2$ and $\alpha \notin \mathbb{Q}$, the function $f(x)=1_{[0, \beta)}(x)-1_{[\beta, 2 \beta)}(x)$ defines an ergodic $\mathbb{Z}$-valued cocycle. This is valid more generally for :

$$
f(x)=\sum_{1 \leq i \leq N} m_{i}\left(1_{\left[0, r_{i}\right)}(x)-1_{\left[r_{i}, 2 r_{i}\right)}(x)\right),
$$

with rationals $\left(r_{i}\right)$ in $(0,1 / 2)$ so that $r_{i} \neq r_{j}$ and $r_{i} \neq 2 r_{j}$ for $i \neq j$, together with integers $\left(m_{i}\right)$ such that $\operatorname{gcd}\left(m_{1}, \cdots, m_{N}\right)=1$. One may also consider :

$$
f(x)=\sum_{k>1} \frac{1}{k^{2}}\left(1_{[0,1 /(2 k))}(x)-1_{[1 /(2 k), 1 / k)}(x)\right),
$$

which defines an ergodic $\mathbb{R}$-valued cocycle for any $\alpha$ with bounded $\left(a_{n}\right)$. The latter cocycle is furthermore $t$-ergodic for all $t \in \mathbb{R}$, for $\mu$-a.-e. $\alpha$.

Remark. - In the second item of the theorem we haven't been able to prove that Maharam measures are the only Radon ergodic ones without using $\rho_{u}$. Unicity is proved in [7] when $\operatorname{supp}(u)$ is finite and $\left(q_{n} \rho_{u}\right)_{n}$ has infinitely many cluster values modulo one. If $\rho_{u}=0$ it is delicate to establish that the non-degeneracy of $U_{n}$ at certain rigid times is "seen" by an arbitrary ergodic Radon measure (this is the first step towards unicity; cf [2]). We also have no example of a non-regular cocycle of this form. This point is discussed later.

Let us give an application to Davenport series $D(x)=\sum_{l>1} c_{l}\{l x\}^{\star}$. Such series appear since a long time in the litterature, as furnishing abundant examples and counter-examples in analysis. Their systematic study was initiated by Davenport [10, 11]; see the presentation by Jaffard [14].

Proposition 4.3. Let $\alpha \notin \mathbb{Q}$ and $D(x)=\sum_{l \geq 1} c_{l}\{l x\}^{\star}$, with $\left(l c_{l}\right)_{l \geq 1} \in l^{1}$. Let $c=\left\{c_{l}\right\}_{l \geq 1}$.

i) If $\sum_{l \geq 1} l c_{l} \neq 0$, then $T_{D}$ is ergodic in $\mathbb{T} \times \mathbb{R}$.

ii) If $\sum_{l \geq 1} l c_{l}=0$, the previous results apply because :

$$
D(x)=U(x)=\sum_{r \in \mathbb{Q}^{*}(\mathbb{T})} u_{r}\left(1_{[0, r)}(x)-r\right),
$$

with $u=\left\{u_{r}\right\}_{r \in \mathbb{Q}^{*}(\mathbb{T})}$ so that $u_{r}=\sum_{m \geq 1} c_{m d(r)}$. Also $\overline{\sigma(u)}=\overline{\sigma(c)}$ and $H_{u}=\overline{\sigma\left(c \cup\left\{\left(\sum c_{l}\right) / 2\right\}\right)}$. 


\subsection{Proof of lemma 4.1}

This is clear for $(C)$. Let us turn to $\left(C^{\prime}\right)$. We shall prove the following stronger result : for all $A>0$ one can find $B>A$ so that along a subsequence $q_{n}$ is prime and $A \leq a_{n+1} \leq B$. We use a difficult result of Erdös [12], theorem II, proving a particular case of the Duffin-Schaeffer conjecture : if $\varepsilon>0$ and $n_{1}<n_{2}<\cdots$ is a sequence verifying $\sum_{i>1} \varphi\left(n_{i}\right) / n_{i}^{2}=+\infty$, then :

$$
\mu\left(\limsup _{i \rightarrow+\infty} A_{i, \varepsilon}\right)=1, \text { where } A_{i, \varepsilon}=\cup_{r \in \mathbb{Q}(\mathbb{T}), d(r)=n_{i}}\left[r-\varepsilon / n_{i}^{2}, r+\varepsilon / n_{i}^{2}\right]
$$

Remark that when $\alpha \in\left[r-\varepsilon / n_{i}^{2}, r+\varepsilon / n_{i}^{2}\right]$ with $d(r)=n_{i}$ and $\varepsilon<1 / 2$ then $r$ has to be some convergent $p_{k} / q_{k}$ of $\alpha$. As $1 /\left(2 q_{k} q_{k+1}\right) \leq\left|\alpha-p_{k} / q_{k}\right|$, we get $a_{k+1}>1 /(4 \varepsilon)$. We take for $\left(n_{i}\right)$ the sequence of primes, which checks the condition because $\sum_{p \in \mathcal{P}} 1 / p=+\infty$. Next, for $\varepsilon>0$ set :

$$
B_{i, \varepsilon}=\cup_{r \in \mathbb{Q}(\mathbb{T}), d(r)=n_{i}}\left[r+\varepsilon /\left(2 n_{i}^{2}\right), r+\varepsilon / n_{i}^{2}\right] .
$$

We have $B_{i, \varepsilon} \subset A_{i, \varepsilon}$ and $\mu\left(B_{i, \varepsilon}\right) \geq \mu\left(A_{i, \varepsilon}\right) / 4$. By a lemma of Cassels [5] :

$$
\mu\left(\limsup _{i \rightarrow+\infty} B_{i, \varepsilon}\right)=\mu\left(\limsup _{i \rightarrow+\infty} A_{i, \varepsilon}\right)=1 .
$$

For $\alpha \in\left[r+\varepsilon /\left(2 n_{i}^{2}\right), r+\varepsilon / n_{i}^{2}\right]$ with $d(r)=n_{i}$, we have $r=p_{k} / q_{k}$ for some $k$ with $a_{k+1} \leq 2 / \varepsilon$, because $\left|\alpha-p_{k} / q_{k}\right|<1 /\left(q_{k} q_{k+1}\right)$. We finally choose $\alpha \in \cap_{m>2} \lim \sup _{i \rightarrow+\infty} B_{i, 1 / m}$.

\subsection{Proof of theorem $4.2 i$ )}

Proposition 4.4. If $U$ is regular, then $\overline{\sigma(u)} \subset \mathcal{E}(U)$.

Proof of the proposition : We adapt [6]. Suppose that $\theta:=U-g+T g \in \mathcal{E}(U), \mu$-a.-e., for a measurable $g$. If $\overline{\sigma(u)} \not \subset \mathcal{E}(U)$, then necessarily $\mathcal{E}(U)=\gamma \mathbb{Z}$ for some $\gamma \geq 0$ and $u_{r_{0}} \notin \mathcal{E}(U)$ for some $r_{0} \in \mathbb{Q}^{*}(\mathbb{T})$. Let $\delta=d\left(u_{r_{0}}, \mathcal{E}(U)\right)>0$ and fix $N>d\left(r_{0}\right)$ so that :

$$
\sum_{r \in \mathbb{Q}^{*}(\mathbb{T}), d(r)>N}\left|u_{r}\right| \leq \delta / 8 .
$$

Let $\varepsilon=1 /\left(32 N^{4}\right)$ and $K \subset \mathbb{T}$ be a compact set with $\mu(K)>1-\varepsilon$ so that $g_{\mid K}$ is uniformly continuous. Choose $\eta>0$ so that for $x, y$ in $K$ :

$$
d(x, y)<\eta \Rightarrow|g(x)-g(y)| \leq \delta / 8 .
$$

Introduce $\Gamma_{n}=\left\{r-k \alpha \mid r \in \mathbb{Q}(\mathbb{T}), d(r) \leq N, 0 \leq k<q_{n} / N^{2}\right\}$ and the corresponding partition $P_{n}$ of $\mathbb{T}$. If $x$ and $x^{\prime}$ are in the same piece of $P_{n}$, then the intervals $\left[x, x^{\prime}\right]+k \alpha$, for $0 \leq k<q_{n} / N^{2}$, meet no $r \in \mathbb{Q}(\mathbb{T})$ with $d(r) \leq N$. They are also disjoint if $d\left(x, x^{\prime}\right)<1 /\left(2 q_{n}\right)$. Therefore, by $(4.1)$ :

$$
\left|U_{q_{n} / N^{2}}(x)-U_{q_{n} / N^{2}}\left(x^{\prime}\right)\right| \leq \sum_{r \in \mathbb{Q}(\mathbb{T}), d(r)>N}\left|u_{r}\right| \leq \delta / 8,
$$

considering $q_{n} / N^{2}$ as an integer. For $0 \leq j \leq q_{n} / N^{2}$, let $\delta_{j}^{-}$and $\delta_{j}^{+}$be the length of the left and right intervals of $r_{0}-j \alpha$ in $P_{n}$. Maybe cutting these intervals, introduce the left and right subintervals $I_{j}^{ \pm}$touching $r_{0}-j \alpha$ and with length $\min \left\{1 /\left(2 q_{n}\right), \delta_{j}^{ \pm} / 2\right\}$.

Fix $0 \leq j \leq q_{n} / N^{2}$ and take points $x \in I_{j}^{-}$and $y \in I_{j}^{+}, \mu$-typical for the property " $T^{n} \theta \in \mathcal{E}(U)$, $n \in \mathbb{Z}$ ". Then :

$$
\begin{aligned}
d\left(u_{r_{0}}, \mathcal{E}(U)\right) & \leq\left|u_{r_{0}}-\theta_{\frac{q_{n}}{N^{2}}}(x)+\theta_{\frac{q_{n}}{N^{2}}}(y)\right| \\
& \leq\left|u_{r_{0}}-U_{\frac{q_{n}}{N^{2}}}(x)+U \frac{q_{n}}{N^{2}}(y)\right|+|g(x)-g(y)|+\left|g\left(x+\frac{q_{n}}{N^{2}} \alpha\right)-g\left(y+\frac{q_{n}}{N^{2}} \alpha\right)\right| .
\end{aligned}
$$


In (4.4) consider the first term on the right. Introducing left and right limits of the ergodic sums at $r_{0}$, by (4.3), it is less than or equal to :

$$
\left|u_{r_{0}}-U_{\frac{q_{n}}{N^{2}}}\left(r_{0}^{-}\right)+U_{\frac{q_{n}}{N^{2}}}\left(r_{0}^{+}\right)\right|+\left|U_{\frac{q_{n}}{N^{2}}}(x)-U_{\frac{q_{n}}{N^{2}}}\left(r_{0}^{-}\right)\right|+\left|U_{\frac{q_{n}}{N^{2}}}(y)-U_{\frac{q_{n}}{N^{2}}}\left(r_{0}^{+}\right)\right| \leq 0+\delta / 8+\delta / 8 .
$$

As a result, $d\left(u_{r_{0}}, \mathcal{E}(U)\right) \leq \delta / 4+|g(x)-g(y)|+\left|g\left(x+q_{n} / N^{2} \alpha\right)-g\left(y+q_{n} / N^{2} \alpha\right)\right|$. If now $x$ and $y$ were both in $K \cap\left(K-q_{n} / N^{2} \alpha\right)$, then $d\left(u_{r_{0}}, \mathcal{E}(U)\right) \leq \delta / 2$, by $(4.2)$, because $1 / q_{n}<\eta$ for large $n$. Hence $\mu$-almost all $I_{j}^{-}$or $\mu$-almost all $I_{j}^{+}$is included in $K^{c} \cup\left(K-q_{n} / N^{2} \alpha\right)^{c}$, giving :

$$
\frac{1}{2}\left(q_{n} / N^{2}\right) \min _{0 \leq j<q_{n} / N^{2}}\left\{1 /\left(2 q_{n}\right), \delta_{j}^{-} / 2, \delta_{j}^{+} / 2\right\} \leq 2 \varepsilon .
$$

Next $\delta_{j}^{ \pm} \geq \min _{(r, k) \neq\left(r_{0}, j\right), d(r) \leq N, 0 \leq k \leq q_{n} / N^{2}}\left\{\left|(r-k \alpha)-\left(r_{0}-j \alpha\right)\right|\right\}$. If $k=j$, then $r \neq r_{0}$ and the related quantity is $\geq 1 / N^{2}$. If $k \neq j$, then :

$$
\left|(r-k \alpha)-\left(r_{0}-j \alpha\right)\right| \geq \frac{1}{d(r) d\left(r_{0}\right)} d\left((k-j) d(r) d\left(r_{0}\right) \alpha, \mathbb{Z}\right) \geq \frac{1}{N^{2}} \frac{1}{2 q_{n}} .
$$

We thus get $1 /\left(8 N^{4}\right) \leq 2 \varepsilon$, contradicting the hypothesis on $\varepsilon$. As a result, $\overline{\sigma(u)} \subset \mathcal{E}(U)$.

LEMMA 4.5. Let $t \in \mathbb{R}$. If $\overline{\sigma(u)} \subset \mathcal{E}_{t}(U)$, then $\mathcal{E}_{t}(U)=H_{u}$.

Proof of the lemma : Supposing that $\rho_{u} \notin \overline{\sigma(u)}$, necessarily $\overline{\sigma(u)}=\gamma \mathbb{Z}$ for some $\gamma>0$ and $u$ has finite support. Write $\rho_{u}=\gamma P / Q,(P, Q)=1$. As $\left(q_{n}, q_{n+1}\right)=1$, fix $a, b$ and $(\psi(p)) \rightarrow+\infty$ so that $a q_{\psi(p)}+b q_{\psi(p)-1}=1 \bmod Q$ for all $p$. We may suppose that $a q_{\psi(p)}+b q_{\psi(p)-1} \rightarrow+\infty$, up to adding some $K Q$ to $a$.

Introduce $U+\overline{\sigma(u)} \in H_{u} / \overline{\sigma(u)}$, the cocycle induced by $U$ in $H_{u} / \overline{\sigma(u)}$. Then $\mu_{t, u}$-a.-e. :

$$
U_{a q_{\psi(p)}}+b q_{\psi(p)-1}+\overline{\sigma(u)}=\left(a q_{\psi(p)}+b q_{\psi(p)-1}\right) \rho_{u}+\overline{\sigma(u)}=\overline{\gamma P / Q}+\overline{\sigma(u)}=\rho_{u}+\overline{\sigma(u)}
$$

By the analogous statement as lemma 3.3 for $H_{u} / \overline{\sigma(u)}$, cf $[\mathbf{1}], \rho_{u}+\overline{\sigma(u)}$ is a $t$-essential value for $U+\overline{\sigma(u)}$ (with the same proof as that of lemma 3.3, using that $a$ and $b$ are fixed, therefore giving in (3.1) an upper-bound of the form $\varepsilon \exp (|t|(|a|+|b|) V(U))$. The rest of the proof is now more or less standard : for all $\varepsilon>0$ and $A \in \mathcal{B}(\mathbb{T})$ with $\mu_{t, u}(A)>0$, there exists $n \in \mathbb{Z}$ so that :

$$
\mu_{t, u}\left(A \cap T^{-n} A \cap\left\{d\left(U_{n}+\overline{\sigma(u)}, \rho_{u}+\overline{\sigma(u)}\right)<\varepsilon\right\}\right)>0 .
$$

There thus exists an integer $k$ so that $\mu_{t, u}\left(A \cap T^{-n} A \cap\left\{d\left(U_{n}, \rho_{u}-k \gamma\right)<\varepsilon\right\}\right)>0$. Call $B$ the set appearing here. As $k \gamma$ is a $t$-essential value for $U$, there exists $m \in \mathbb{Z}$ so that :

$$
\mu_{t, u}\left(B \cap T^{-m} B \cap\left\{d\left(U_{m}, k \gamma\right)<\varepsilon\right\}\right)>0 .
$$

The set involved above is included in $A \cap T^{-m-n} A$ and on this set $d\left(U_{m+n}, \rho_{u}\right)<2 \varepsilon$, because $d\left(U_{m}, k \gamma\right)<\varepsilon$ and $d\left(U_{n} \circ T^{m}, \rho_{u}-k \gamma\right)<\varepsilon$. Hence :

$$
\mu_{t, u}\left(A \cap T^{-n-m} A \cap\left\{d\left(U_{n+m}, \rho_{u}\right)<2 \varepsilon\right\}\right)>0 .
$$

As a result $\rho_{u}$ is a $t$-essential value for $U$ and $\mathcal{E}_{t}(U)=H_{u}$.

Proposition 4.6. If $(C)$ is true or $\operatorname{supp}(u)$ is finite, then $U$ is regular. 
Proof of the proposition : i) First, by (1.4) and (1.3) :

$$
U_{n}(x)=-\frac{1}{2 i \pi} \sum_{m \neq 0} \frac{e^{2 i \pi m x}}{m}\left(\frac{e^{2 i \pi m n \alpha}-1}{e^{2 i \pi m \alpha}-1}\right) \xi(m), \xi(m)=\sum_{r \in \mathbb{Q}(\mathbb{T})} v_{r} e^{-2 i \pi m r} .
$$

Notice that $\xi$ is an almost-periodic function on $\mathbb{N}$. For some constant $C>0$ :

$$
\left\|U_{n}\right\|_{2}^{2} \geq C \sum_{m \geq 1} \frac{((m n \alpha))^{2}}{m^{2}((m \alpha))^{2}}|\xi(m)|^{2}
$$

ii) We next make another computation. Let $I_{1, n}=\left[p_{n} / q_{n}, \alpha\right]$ be the small interval on $\mathbb{T}$ determined by $p_{n} / q_{n}$ and $\alpha$ and $I_{k, n}=\left[k p_{n} / q_{n}, k \alpha\right]$ its image by $\times k$. Introduce the disjoint union $J_{n}=$ $\cup_{0 \leq k<q_{n}} I_{k, n}$ and set $\varepsilon_{n}=\operatorname{sign}\left(\alpha-p_{n} / q_{n}\right)$. By (1.5) :

$$
\begin{aligned}
\{.\}_{q_{n}}^{\star}(x) & =\sum_{0 \leq k<q_{n}}\left\{x+k p_{n} / q_{n}\right\}^{\star}+\sum_{0 \leq k<q_{n}}\{x+k \alpha\}^{\star}-\left\{x+k p_{n} / q_{n}\right\}^{\star} \\
& =\left\{q_{n} x\right\}^{\star}+\frac{q_{n}\left(q_{n}-1\right)}{2}\left(\alpha-p_{n} / q_{n}\right)-\varepsilon_{n} \sum_{0 \leq k<q_{n}} 1_{-x \in I_{k, n}} .
\end{aligned}
$$

As a result, using that $\sum_{r \in \mathbb{Q}(\mathbb{T})} v_{r}=0$ :

$$
\begin{aligned}
U_{q_{n}}(x) & =\sum_{r \in \mathbb{Q}(\mathbb{T})} v_{r}\left\{q_{n}(x-r)\right\}^{\star}-\varepsilon_{n} \sum_{r \in \mathbb{Q}(\mathbb{T})} v_{r} 1_{-x \in J_{n}-r} \\
& =\sum_{r \in \mathbb{Q}^{*}(\mathbb{T})} u_{r}\left(1_{\left[0,\left\{q_{n} r\right\}\right)}\left(\left\{q_{n} x\right\}\right)-\left\{q_{n} r\right\}\right)-\varepsilon_{n} \sum_{r \in \mathbb{Q}^{*}(\mathbb{T})} u_{r}\left(1_{-x \in J_{n}-r}-1_{-x \in J_{n}}\right) .
\end{aligned}
$$

Setting $\gamma_{r}(n)=\sum_{s \in \mathbb{Q}^{*}(\mathbb{T}), q_{n} s=r} u_{s}$, we obtain :

$$
U_{q_{n}}(x)=\sum_{r \in \mathbb{Q}^{*}(\mathbb{T})} \gamma_{r}(n)\left(1_{[0, r)}\left(\left\{q_{n} x\right\}\right)-r\right)-\varepsilon_{n} \sum_{r \in \mathbb{Q}^{*}(\mathbb{T})} u_{r}\left(1_{-x \in J_{n}-r}-1_{-x \in J_{n}}\right)
$$

iii) Suppose now that $U \neq 0$. Let $m_{0}$ be such that $\xi\left(m_{0}\right) \neq 0$ and fix $N$ with :

$$
\left|\sum_{r \in \mathbb{Q}(\mathbb{T}), d(r) \leq N} u_{r} e^{-2 i \pi m_{0} r}\right|>\frac{3}{4}\left|\xi\left(m_{0}\right)\right| \text { and } \sum_{r \in \mathbb{Q}(\mathbb{T}), d(r)>N}\left|u_{r}\right|<\frac{1}{4}\left|\xi\left(m_{0}\right)\right| .
$$

Let integers $a, b$ and $(\psi(p)) \rightarrow+\infty$ be so that $m_{p}:=a q_{\psi(p)}+b q_{\psi(p)-1}$ verifies $m_{p}=m_{0} \bmod N$ ! for all $p$. We can suppose that $|b|<N$ ! and $K N$ ! $\leq a<2 K N$ !, with $K \geq 1$ specified later. Notice that $m_{p}\left(\left(m_{p} \alpha\right)\right) \leq(a+|b|)^{2}$.

Using the term of index $m_{p}$ in $(4.5)$ and $\left(\left(n m_{p} \alpha\right)\right) \geq\left|\left(\left(n a q_{\psi(p)} \alpha\right)\right)-\left(\left(n b q_{\psi(p)-1} \alpha\right)\right)\right|$, we obtain that for all integers $n, p$ :

$$
\left\|U_{n}\right\|_{2}^{2} \geq \frac{C}{(a+|b|)^{4}} \frac{\left|\xi\left(m_{0}\right)\right|^{2}}{4}\left[\left(\left(n a q_{\psi(p)} \alpha\right)\right)-\left(\left(n b q_{\psi(p)-1} \alpha\right)\right)\right]^{2} .
$$

- In a first case, suppose that $a_{n} \leq M$ for all $n$. Take $K=8 M$ and $k_{0}$ so that $(3 / 2)^{k_{0}}>$ $20 M N$ !. For large $p$ and $n=q_{\psi(p)-k_{0}}$, we have $\left(\left(n a q_{\psi(p)} \alpha\right)\right)=n a\left(\left(q_{\psi(p)} \alpha\right)\right)$ and $\left(\left(n b q_{\psi(p)-1} \alpha\right)\right) \leq$ $n|b|\left(\left(q_{\psi(p)-1} \alpha\right)\right)$. Thus, for large $p,\left|\left(\left(n a q_{\psi(p)} \alpha\right)\right)-\left(\left(n b q_{\psi(p)-1} \alpha\right)\right)\right|$ is larger than or equal to :

$$
q_{\psi(p)-k_{0}}\left(a\left(\left(q_{\psi(p)} \alpha\right)\right)-|b|\left(\left(q_{\psi(p)-1} \alpha\right)\right)\right) \geq q_{\psi(p)-k_{0}}\left(\left(q_{\psi(p)} \alpha\right)\right)(a-4|b| M) \geq \frac{a}{2} \frac{1}{(2 M)^{k_{0}+1}} .
$$

By Denjoy-Koksma's inequality, $\left\|U_{q_{\psi(p)-k_{0}}}\right\|_{\infty} \leq 2 \sum_{r \in \mathbb{Q}(\mathbb{T})}\left|u_{r}\right|$, so $\left(\mathcal{L}\left(U_{q_{\psi(p)-k_{0}}}\right)\right)_{p}$ is tight. Any cluster value in law is not zero because, by $(4.8),\left\|U_{q_{\psi(p)-k_{0}}}\right\|_{2}$ is bounded from below by a positive 
constant. As $\left(q_{\psi(p)-k}\right)_{p}$ is a rigid sequence, there exists a non-zero finite essential value for $U$, by lemma 3.3. Therefore $U$ is regular.

- Suppose next that for all $A>0$ there exist $B$ and $(\psi(p)) \rightarrow+\infty$ so that $A \leq a_{\psi(p)+1} \leq B$. Take $n=q_{\psi(p)}$ in (4.8), $K=4$ and $A=4 N$ !. Then :

$$
\left(\left(n a q_{\psi(p)} \alpha\right)\right)-\left(\left(n b q_{\psi(p)-1} \alpha\right)\right) \geq q_{\psi(p)}\left(\left(q_{\psi(p)} \alpha\right)\right)(a-|b|) \geq \frac{a}{8 B} .
$$

We next conclude in the same way.

- Suppose now $\left(a_{n}\right)$ unbounded. Let us place on a subsequence where $a_{n+1} \rightarrow+\infty$ and define :

$$
K_{n}=\cup_{r \in \mathbb{Q}(\mathbb{T}), d(r)<\min \left\{q_{n}, a_{n+1}^{1 / 4}\right\}}\left(J_{n}-r\right) .
$$

Decomposing the last term of (4.7), we obtain that uniformly in $x \notin-K_{n}$ :

$$
U_{q_{n}}(x)=\sum_{r \in \mathbb{Q}^{*}(\mathbb{T})} \gamma_{r}(n)\left(1_{[0, r)}\left(\left\{q_{n} x\right\}\right)-r\right)+o(1) .
$$

As $\mu\left(J_{n}\right) \leq 1 /\left(2 a_{n+1}\right)$ we have $\mu\left(K_{n}\right) \leq a_{n+1}^{1 / 2} \mu\left(J_{n}\right) \leq 1 /\left(2 a_{n+1}^{1 / 2}\right) \rightarrow 0$. By Slutsky's lemma on the convergence of random variables, the behaviour in distribution of $\left(U_{q_{n}}\right)_{n \geq 1}$ along the considered subsequence is that of :

$$
\sum_{r \in \mathbb{Q}^{*}(\mathbb{T})} \gamma_{r}(n)\left(1_{[0, r)}\left(\left\{q_{n} x\right\}\right)-r\right)={ }_{\mathcal{L}} \sum_{r \in \mathbb{Q}^{*}(\mathbb{T})} \gamma_{r}(n)\left(1_{[0, r)}(x)-r\right) .
$$

- Suppose that for some $r_{0} \in \mathbb{Q}^{*}(\mathbb{T}),\left(\gamma_{r_{0}}(n)\right)$ does not tend to 0 . Then there exists a constant $\gamma_{r_{0}} \neq 0$ so that $\gamma_{r_{0}}(n) \rightarrow \gamma_{r_{0}}$ along a subsequence. Using the diagonal procedure, we construct $\left(\gamma_{r}\right)_{r \in \mathbb{Q}^{*}(\mathbb{T})} \in l^{1}$ and get a cluster value in law for the narrow topology of the form $\mathcal{L}(X)$, with :

$$
X=\sum_{r \in \mathbb{Q}^{*}(\mathbb{T})} \gamma_{r}\left(1_{[0, r)}(x)-r\right)
$$

Clearly both $X\left(r_{0}^{-}\right)$and $X\left(r_{0}^{+}\right)$belong to $\operatorname{supp}(\mathcal{L}(X))$ and thus to $\mathcal{E}(U)$ by lemma 3.3. As $\mathcal{E}(U)$ is a group, $0 \neq \gamma_{r_{0}}=X\left(r_{0}^{-}\right)-X\left(r_{0}^{+}\right) \in \mathcal{E}(U)$. As a result $U$ is regular.

- Suppose now that for each $r, \gamma_{r}(n) \rightarrow 0$. When $\operatorname{supp}(u)$ is finite, necessarily $\gamma_{r}(n)=0$ for $n$ large enough. Hence, for $n$ large enough, by $(4.7), U_{q_{n}}(x)=-\varepsilon_{n} \sum_{r \in \mathbb{Q}(\mathbb{T})} v_{r} 1_{-x \in J_{n}-r}$. The $\left(J_{n}+m q_{n} \alpha\right)_{0 \leq m<a_{n+1}}$ are disjoint, so :

$$
U_{m q_{n}}(x)=-\varepsilon_{n} \sum_{r \in \mathbb{Q}(\mathbb{T})} v_{r} 1_{-x \in \cup_{0 \leq u<m} J_{n}+u q_{n} \alpha-r},
$$

for $0 \leq m<a_{n+1}$. In (4.8), with $K=4, m_{p}=a_{\psi(p)+1} /(10 N !)$ and $n=m_{p} q_{\psi(p)}$, we get :

$$
\left(\left(n a q_{\psi(p)} \alpha\right)\right)-\left(\left(n b q_{\psi(p)-1} \alpha\right)\right) \geq m_{p} q_{\psi(p)}\left(\left(q_{\psi(p)} \alpha\right)\right)(a-|b|) \geq \frac{a}{40 N !} .
$$

Hence $\left(U_{m_{p} q_{\psi(p)}}\right)_{p}$ is uniformly bounded and bounded from below by a positive constant in $L^{2}(\mu)$, by (4.8). As $m_{p} q_{\psi(p)} \alpha \rightarrow 0 \bmod 1$, we conclude again with lemma 3.3 that $U$ is regular.

\subsection{Proof of theorem $4.2 \mathrm{ii})$}

Let $n \geq 1$. Consider the $(k \alpha)_{0 \leq k<q_{n}}$ on $\mathbb{T}$ and denote by $p_{k}(n) \alpha$ the predecessor of $k \alpha$ for the trigonometric ordering, with $0<p_{k}(n) \leq q_{n}$. As a first remark, $\left\{q_{n} x\right\}^{\star}$ is linear with slope $q_{n}$ going from $-1 / 2$ to $1 / 2$ on each interval $\left(l / q_{n},(l+1) / q_{n}\right)$. It can therefore be rewritten as :

$$
\left\{q_{n} x\right\}^{\star}=\sum_{0 \leq k<q_{n}}\left(q_{n}\left(x+k p_{n} / q_{n}\right)-1 / 2\right) 1_{x \in\left[-k p_{n} / q_{n},-p_{k}(n) p_{n} / q_{n}\right]} .
$$


Without loss of generality, suppose now that $\varepsilon_{n}=+1$, ie $\alpha>p_{n} / q_{n}$. We next have :

$$
\left\{q_{n} x\right\}^{\star}-\sum_{0 \leq k<q_{n}} 1_{x \in\left[-k \alpha,-k p_{n} / q_{n}\right]}=\sum_{0 \leq k<q_{n}}\left(q_{n}\left(x+k p_{n} / q_{n}\right)-1 / 2\right) 1_{x \in\left[-k \alpha,-p_{k}(n) \alpha\right]},
$$

as (make a picture) this operation of subtraction moves the domain of each linear part $q_{n}(x+$ $\left.k p_{n} / q_{n}\right)-1 / 2$ from $\left(-k p_{n} / q_{n},-p_{k}(n) p_{n} / q_{n}\right)$ to $\left(-k \alpha,-p_{k}(n) \alpha\right)$. By (4.6), we obtain :

$$
\{\cdot\}_{q_{n}}^{\star}(x)=\sum_{0 \leq k<q_{n}}\left(q_{n}\left(x+k p_{n} / q_{n}\right)-1 / 2\right) 1_{\left[-k \alpha,-p_{k}(n) \alpha\right]}(x)+\frac{q_{n}\left(q_{n}-1\right)}{2}\left(\alpha-p_{n} / q_{n}\right) .
$$

By (1.4) and as the $v_{r}$ sum to 0 :

$$
U_{q_{n}}(x)=\sum_{r \in \mathbb{Q}(\mathbb{T})} v_{r} \sum_{0 \leq k<q_{n}}\left(q_{n}\left(x-r+k p_{n} / q_{n}\right)-1 / 2\right) 1_{\left[-k \alpha,-p_{k}(n) \alpha\right]}(x-r) .
$$

For $\delta>0$, let $A^{+}(n, \delta)=\cup_{0 \leq k<q_{n}}\left[0, \delta / q_{n}\right]-k \alpha, A^{-}(n, \delta)=\cup_{0 \leq k<q_{n}}\left[-\delta / q_{n}, 0\right]-k \alpha$. Set finally $B(n, \delta)=A^{+}(n, \delta) \cup A^{-}(n, \delta)$. If $x \in(B(n, \delta)+r) \cap\left(B(n, \delta)+r^{\prime}\right)$ for $r \neq r^{\prime}$ in $\mathbb{Q}(\mathbb{T})$, then :

$$
r-r^{\prime}=\left(k_{n}-k_{n}^{\prime}\right) \alpha+y_{n}^{\prime}-y_{n},
$$

for integers $0 \leq k_{n}, k_{n}^{\prime}<q_{n}$ and reals $y_{n}, y_{n}^{\prime}$ with $\left|y_{n}\right|,\left|y_{n}^{\prime}\right| \leq \delta / q_{n}$. If the prime factors of $q_{n}$ are large enough, then $\left(\left(q_{n}\left(r-r^{\prime}\right)\right)\right) \geq 1 /\left(d(r) d\left(r^{\prime}\right)\right)$. Multiplying (4.11) by $q_{n}$ and taking $d(., \mathbb{Z})$ :

$$
\frac{1}{d(r) d\left(r^{\prime}\right)} \leq\left(\left(q_{n}\left(r-r^{\prime}\right)\right)\right) \leq \frac{1}{a_{n+1}}+2 \delta .
$$

Let $t \in \mathbb{R}$. As before, fixing $r_{0} \in \mathbb{Q}^{*}(\mathbb{T})$, it is enough to show that $u_{r_{0}} \in \mathcal{E}_{t}(U)$. Fix $\varepsilon>0$ and choose an integer $N>\max \left\{d\left(r_{0}\right), 1 /\left(3 \varepsilon d\left(r_{0}\right)\right\}\right.$ verifying :

$$
\sum_{r \in \mathbb{Q}(\mathbb{T}), d(r)>N}\left|v_{r}\right|<\varepsilon
$$

Let $\delta=1 /\left(3 N d\left(r_{0}\right)\right)$ and $A=100 N d\left(r_{0}\right)$. Take $B \geq A$ and a subsequence of $\left(q_{n}\right)$ where the smallest prime factor of $q_{n}$ tends to infinity, together with $A \leq a_{n+1} \leq B$. By (4.12), both $A^{ \pm}(n, \delta)+r_{0}$ are disjoint from the $B(n, \delta)+r$, for $r \in \mathbb{Q}(\mathbb{T})$ with $r \neq r_{0}$ and $d(r) \leq N$.

Let $y=-k_{n} \alpha+y_{n} \in A^{+}(n, \delta), 0 \leq k_{n}<q_{n}, 0 \leq y_{n}<\delta / q_{n}$. Set $x=y+r_{0}$. As $x-r_{0}=y \in$ $\left(-k_{n} \alpha,-p_{k_{n}}(n) \alpha\right)$, the term corresponding to $r_{0}$ in (4.10) is :

$$
\begin{aligned}
\sum_{0 \leq k<q_{n}}\left(q_{n}\left(x-r_{0}+k p_{n} / q_{n}\right)-1 / 2\right) 1_{\left[-k \alpha,-p_{k}(n) \alpha\right]}\left(x-r_{0}\right) & =q_{n}\left(-k_{n} \alpha+y_{n}+k_{n} p_{n} / q_{n}\right)-1 / 2 \\
& =-\varepsilon_{n} k_{n}\left(\left(q_{n} \alpha\right)\right)+q_{n} y_{n}-1 / 2 \\
& =-1 / 2+r_{n}(x),
\end{aligned}
$$

with $\left|r_{n}(x)\right| \leq \delta+1 / a_{n+1} \leq 1 /\left(2 N d\left(r_{0}\right)\right)$. Consider next $r \in \mathbb{Q}(\mathbb{T})$ with $r \neq r_{0}$ and $d(r) \leq N$. From the previous discussion, we know that $\left(B(n, \delta)+r_{0}\right) \cap(B(n, \delta)+r)=\varnothing$. As $x-r_{0} \in$ $B(n, \delta)$, we have $x-r \notin B(n, \delta)$ and so clearly $x-r \notin \cup_{0 \leq k<q_{n}}\left[-k \alpha,-k p_{n} / q_{n}\right]$. Therefore for the corresponding term in (4.10), using also (4.9) at the end, we can write :

$$
\begin{aligned}
& \sum_{0 \leq k<q_{n}}\left(q_{n}\left(x-r+k p_{n} / q_{n}\right)-1 / 2\right) 1_{\left[-k \alpha,-p_{k}(n) \alpha\right]}(x-r) \\
= & \sum_{0 \leq k<q_{n}}\left(q_{n}\left(x-r+k p_{n} / q_{n}\right)-1 / 2\right) 1_{\left[-k p_{n} / q_{n},-p_{k}(n) p_{n} / q_{n}\right]}(x-r) \\
= & \left\{q_{n}(x-r)\right\}^{\star}=\left\{s_{n}(x)+\left(r_{0}-r\right) q_{n}\right\}^{\star}
\end{aligned}
$$

with $s_{n}(x)=q_{n}\left(y_{n}-k_{n} \alpha\right)$ and thus $\left(\left(s_{n}(x)\right)\right)=\left(\left(q_{n} y_{n}-k_{n} q_{n} \alpha\right)\right) \leq \delta+1 / a_{n+1} \leq 1 /\left(2 N d\left(r_{0}\right)\right)$. 
Take another subsequence on which $\left(r_{0}-r\right) q_{n}=i_{r} / j_{r} \bmod 1$, with $\left|j_{r}\right| \leq d\left(r_{0}\right) d(r)$, for all $r \in$ $\mathbb{Q}(\mathbb{T})$ with $r \neq r_{0}$ and $d(r) \leq N$. Necessarily $i_{r} / j_{r} \neq 0 \bmod 1$, for all $r$. Set :

$$
K_{r_{0}}=\sum_{r \in \mathbb{Q}(\mathbb{T}), d(r) \leq N, r \neq r_{0}} v_{r}\left\{i_{r} / j_{r}\right\}^{\star} .
$$

Along this subsequence, in (4.10), using (4.13), (4.14), (4.15) and Denjoy-Koksma's inequality as well as that $\left\{s_{n}(x)+i_{r} / j_{r}\right\}^{\star}-\left\{i_{r} / j_{r}\right\}^{\star}=s_{n}(x)$ for $r \neq r_{0}$ and $d(r) \leq N$, we have for all $x \in$ $A^{+}(n, \delta)+r_{0}$,

$$
\begin{aligned}
\left|U_{q_{n}}(x)-K_{r_{0}}+v_{r_{0}} / 2\right| & \leq\left|\sum_{r \in \mathbb{Q}(\mathbb{T}), d(r)>N} v_{r}\{\cdot\}_{q_{n}}^{\star}(x-r)\right|+\left|v_{r_{0}}\left(\{.\}_{q_{n}}^{\star}\left(x-r_{0}\right)+1 / 2\right)\right| \\
& +\left|\sum_{r \in \mathbb{Q}(\mathbb{T}), d(r) \leq N, r \neq r_{0}} v_{r}\left(\{.\}_{q_{n}}^{\star}(x-r)-\left\{i_{r} / j_{r}\right\}^{\star}\right)\right| \\
& \leq 2 \varepsilon+\frac{1}{2 N d\left(r_{0}\right)} \sum_{r \in \mathbb{Q}(\mathbb{T}), d(r) \leq N}\left|v_{r}\right| \leq \varepsilon^{\prime},
\end{aligned}
$$

with $\varepsilon^{\prime}=\varepsilon\left(2+\|v\|_{l^{1}}\right)$. Idem, along the same subsequence, for all $\mathrm{x}$ in $A^{-}(n, \delta)+r_{0}$ :

$$
\left|U_{q_{n}}(x)-K_{r_{0}}-v_{r_{0}} / 2\right| \leq \varepsilon^{\prime} .
$$

As $U_{q_{n}}$ is bounded because of Denjoy-Koksma's inequality, take a cluster value $m$ of $\left(\mathcal{L}_{t}\left(U_{q_{n}}\right)\right)_{n}$ for the narrow topology along the previous subsequence. Then, from (4.16) :

$$
m\left(\bar{B}\left(K_{r_{0}}-v_{r_{0}} / 2, \varepsilon^{\prime}\right)\right) \geq \limsup _{n \in \text { subseq }} \mu_{t, u}\left(U_{q_{n}} \in \bar{B}\left(K_{r_{0}}-v_{r_{0}} / 2, \varepsilon^{\prime}\right)\right) \geq \limsup _{n \in \text { subseq }} \mu_{t, u}\left(A^{+}(n, \delta)+r_{0}\right) .
$$

We will show later that for a constant $C>0$, along the subsequence :

$$
\mu_{t, u}\left(A^{ \pm}(n, \delta)+r_{0}\right) \geq C>0 .
$$

This gives $\bar{B}\left(K_{r_{0}}-v_{r_{0}} / 2, \varepsilon^{\prime}\right) \cap \operatorname{supp}(m) \neq \varnothing$. By lemma $3.3, \bar{B}\left(K_{r_{0}}-v_{r_{0}} / 2, \varepsilon^{\prime}\right) \cap \mathcal{E}_{t}(U) \neq \varnothing$. Similarly one gets $\bar{B}\left(K_{r_{0}}+v_{r_{0}} / 2, \varepsilon^{\prime}\right) \cap \mathcal{E}_{t}(U) \neq \varnothing$. As $\mathcal{E}_{t}(U)$ is a group, $\bar{B}\left(v_{r_{0}}, 2 \varepsilon^{\prime}\right) \cap \mathcal{E}_{t}(U) \neq \varnothing$. This is true for all $\varepsilon>0$ and $\mathcal{E}_{t}(U)$ is closed, so $v_{r_{0}} \in \mathcal{E}_{t}(U)$. This is the desired conclusion.

It remains to show (4.17). We consider $A^{+}(n, \delta)+r_{0}$, the other case being similar. Notice first that from (1.1), for $t \in \mathbb{R}, n \in \mathbb{Z}$ and $A \in \mathcal{B}(\mathbb{T})$ :

$$
\mu_{t, u}\left(T^{-n} A\right)=\int_{A} e^{-t U_{-n}} d \mu_{t, u} .
$$

Define $C_{n}=\left[-\left(\left(q_{n} \alpha\right)\right),\left(\left(q_{n} \alpha\right)\right)\right]$ and $D_{n}=\cup_{0 \leq k<q_{n}} T^{-k} C_{n}+r_{0}$. As $T^{q_{n}} D_{n} \subset A^{+}(n, \delta)+r_{0}$, using next (4.18), we get :

$$
\mu_{t, u}\left(A^{+}(n, \delta)+r_{0}\right) \geq \mu_{t, u}\left(T^{q_{n}} D_{n}\right) \geq e^{-|t| V(U)} \mu_{t, u}\left(D_{n}\right) .
$$

On the other hand, the $\left(T^{m q_{n}} D_{n}\right)_{0 \leq m<2 a_{n+1}}$ cover $\mathbb{T}$. Therefore, using again (4.18) :

$$
1 \leq \mu_{t, u}\left(\cup_{0 \leq m<2 a_{n+1}} T^{m q_{n}} D_{n}\right) \leq \mu_{t, u}\left(D_{n}\right) \sum_{0 \leq m<2 a_{n+1}} e^{|t| m V(U)} .
$$

We conclude that $\mu_{t, u}\left(A^{+}(n, \delta)+r_{0}\right) \geq 1 / \sum_{1 \leq m \leq 2 B+1} e^{|t| m V(U)}>0$. This shows (4.17) and completes the proof of the result.

Remark. - A similar result is true if $\operatorname{supp}(u) \subset\left\{r \in \mathbb{Q}^{*}(\mathbb{T}), d(r) \in \mathcal{P}\right\}$ and $\alpha$ verifies : $\forall A>0 \exists B \geq$ $A \exists(\psi(n)) \rightarrow+\infty$ so that $A \leq a_{\psi(n)}, a_{\psi(n)+1} \leq B$, this time immediately seen to true $\mu$-a.-e.. 
Remark. - For a given $\alpha \notin \mathbb{Q}$, it is very difficult in general to determine the decomposition in prime factors of the denominators of its convergents. Recall that it is a delicate question even for a sequence satisfying a fixed linear recursive relation, a famous open question being for example whether the Fibonacci sequence contains infinitely many primes.

Remark. - The denominators $q_{n}$ and $q_{n+1}$ are always relatively prime, but, as mentioned in [12], for distinct primes $p$ and $q$, there exists $\alpha$ so that $q_{2 n}=p^{u_{n}}$ and $q_{2 n+1}=q^{v_{n}}$. Here is a way of building such an $\alpha$. Writing $\alpha=\left[a_{1}, a_{2}, \cdots\right]$, we have $q_{0}=1$. Choose $a_{1}=q_{1}=q$. Recursively, use first the little Fermat theorem to write $q^{\varphi\left(p^{u_{n}}\right)-1}=l_{n+1} p^{u_{n}}+1$ and next take $a_{n+1}=l_{n+1} q^{v_{n-1}}$ and $q_{2 n+1}=q^{\varphi\left(p^{u_{n}}\right)-1+v_{n-1}}$ in order to satisfy $q_{2 n+1}=a_{n+1} p^{u_{n}}+q^{v_{n-1}}$. For this $\alpha$, an interesting example is as follows. Let $\left(b_{k}\right)_{k \geq 1}$ and $\left(c_{l}\right)_{l \geq 1}$ in $l^{1}$ with $\sum_{k \geq 1} b_{k}=\sum_{l \geq 1} c_{l}=0$. Define :

$$
D(x)=\sum_{k, l \geq 1} b_{k} c_{l}\left\{x+1 / p^{k}+1 / q^{l}\right\}^{\star} .
$$

Following the same ideas one obtains rather easily that $\left(D_{q_{n}}\right)$ converges to zero $\mu$-a.-e., if $\alpha$ is chosen enough Liouville. Is it possible to get a non-regular cocycle in this way ?

\subsection{Proof of proposition 4.3}

Set $u_{r}=C_{d(r)}$, where $C_{q}=\sum_{m \geq 1} c_{m q}, q \geq 1$. As $\left(l c_{l}\right) \in l^{1}$, we get $\left(\varphi(q) C_{q}\right) \in l^{1}$, hence $\left(u_{r}\right)_{r \in \mathbb{Q}^{*}(\mathbb{T})} \in l^{1}$. Now, by $(1.5)$ and using the dominated convergence theorem :

$$
D(x)=\sum_{l \geq 1} c_{l} \sum_{0 \leq k<l}\{x+k / l\}^{\star}=\sum_{r \in \mathbb{Q}(\mathbb{T})} C_{d(r)}\{x+r\}^{\star} .
$$

From the properties of Euler's Totient function $\varphi$ we obtain :

$$
\sum_{r \in \mathbb{Q}(\mathbb{T})} u_{r}=\sum_{q \geq 1} \varphi(q) \sum_{m \geq 1} c_{m q}=\sum_{l \geq 1} c_{l} \sum_{q \mid l} \varphi(q)=\sum_{l \geq 1} l c_{l} .
$$

i) Suppose that $\sum_{l \geq 1} l c_{l} \neq 0$. For $N \geq 1$ fixed later define :

$$
E(x)=\sum_{r \in \mathbb{Q}(\mathbb{T}), d(r)<N} C_{d(r)}\{x+r\}^{\star} \text { and } F=D-E .
$$

Let us respectively compute the sum of the jumps of $E$ and the variation of $F$ :

$$
J(E)=-\sum_{r \in \mathbb{Q}(\mathbb{T}), d(r)<N} C_{d(r)} \text { and } V(F)=\left|\sum_{r \in \mathbb{Q}(\mathbb{T}), d(r) \geq N} C_{d(r)}\right|+\sum_{r \in \mathbb{Q}(\mathbb{T}), d(r) \geq N}\left|C_{d(r)}\right| .
$$

Since $\sum_{l \geq 1} l c_{l} \neq 0$, we have $V(F)<|J(E)|$ for $N$ large enough. By theorem 3 in $[\mathbf{1 7}]$, giving a perturbation result, $D=E+F$ defines an ergodic extension.

ii) Suppose that $\sum_{l \geq 1} l c_{l}=0$. It is sufficient to show that :

$$
\overline{\sigma\left(\left\{C_{q}\right\}_{q \geq 1}\right)}=\overline{\sigma\left(\left\{c_{l}\right\}_{l \geq 1}\right)} \text { and } \sum_{r \in \mathbb{Q}^{*}(\mathbb{T})} r C_{d(r)}=-\left(\sum_{l \geq 1} c_{l}\right) / 2 .
$$

The last point follows from $D\left(0^{+}\right)=-\left(\sum_{l \geq 1} c_{l}\right) / 2=\sum_{r \in \mathbb{Q}(\mathbb{T})} C_{d(r)}$. For the first one, " $\subset$ " is clearly true. For the other direction we use a sieve argument of Haar (cf chap. 1, no. 129, Pólya and Szegö [19]). Let $l \geq 1$. Take $M \geq 1$ and write $p_{1}<\cdots<p_{M}$ for the first $M$ primes. The sum :

$$
\sum_{m \geq 1} c_{m l}-\sum_{i=1}^{M} \sum_{m \geq 1} c_{m l p_{i}}+\sum_{1 \leq i<j \leq M} \sum_{m \geq 1} c_{m l p_{i} p_{j}}+\cdots+(-1)^{M} \sum_{m \geq 1} c_{m l p_{1} \cdots p_{M}}
$$

belongs to $\overline{\sigma\left(\left\{c_{l}\right\}_{l \geq 1}\right)}$ and equals $\sum_{m>1} c_{m l}\left(1-1_{\left\{\exists 1 \leq i \leq M, p_{i} \mid m\right\}}\right)$. Letting $M \rightarrow+\infty$ and using the dominated convergence theorem, the limit is $c_{l}$. As $l$ is arbitrary, this completes the proof of (4.19). 
Remark. - The argument in $i$ ) does not work when $\sum_{l \geq 1} l c_{l}=0$. In this case $V(F) \geq 2|J(E)|$.

Remark. - Following the proof of theorem 4.2, one can show the following property resonant with (1.5) for BV Davenport series : for $\alpha$ in a set of full $\mu$-measure independent of $D,\left(\mathcal{L}\left(D_{q_{n}}\right)\right)_{n \geq 1}$ admits $\mathcal{L}(D)$ as cluster value for the narrow topology.

Remark. - For example proposition 4.3 implies that Hecke's function $\mathcal{H}_{s}(x)=\sum_{l \geq 1} l^{-s}\{l x\}^{\star}$, with $s>2$, defines an ergodic skew-product for any $\alpha \notin \mathbb{Q}$.

Remark. - It would be interesting to replace $\{x\}^{\star}$ by $\{x\}_{\kappa}^{\star}=\sum_{m>1} m^{-\kappa} \sin (2 \pi m x), 0<\kappa<1$. The function $\{x\}_{\kappa}^{\star}$ is continuous on $(0,1)$ but presents a singularity like $1 / x^{1-\kappa}$ at 0 , giving $\{x\}_{\kappa}^{\star} \in$ $L^{p}(\mathbb{T}, \mu)$ for $p<(1-\kappa)^{-1}$ only. Observe that this time for all $q \geq 1$ we have :

$$
\sum_{0 \leq k<q}\{x+k / q\}_{\kappa}^{\star}=q^{1-\kappa}\{q x\}_{\kappa}^{\star}, x \in \mathbb{T}
$$

Setting $U(x)=\sum_{r \in \mathbb{Q}(\mathbb{T})} v_{r}\{x+r\}_{\kappa}^{\star}$, with $\left(v_{r}\right) \in l^{1}$, is $\left(\mathcal{L}\left(U_{q_{n}} / q_{n}^{1-\kappa}\right)\right)_{n \geq 1}$ tight ?

\section{References}

1. Aaronson, J., An introduction to infinite ergodic theory, Mathematical surveys and monographs 50, AMS, Providence, 1997.

2. Aaronson, J., Nakada, H., Sarig, O. and Solomyak, R., Invariant measures and asymptotics for some skew-products, Israël Journal of Math., vol. 128 (2002), 93-134.

3. BRÉmont, J., Ergodic non-abelian smooth extensions of an irrational rotation, Journal of the London Mathematical Society, 2010, 81: 457-476.

4. Brémont, J., Davenport series and almost-sure convergence, Quarterly Journal of Mathematics 62 (2011), no. 4, 825-843.

5. Cassels, J., Some metrical theorems in Diophantine approximation, Proc. Cambridge Phil. Soc, 46 (1950), 209-218.

6. Conze, J.-P., Ergodicité d'une transformation cylindrique, Bulletin de la S. M. F., 108 (1980), $441-456$.

7. Conze, J.-P., Recurrence, ergodicity and invariant measures for cocycles over a rotation, Contemporary Mathematics, vol. 485 (2009), 45-70.

8. Conze, J.-P. And GuivarC'H, Y., Marches en milieu aléatoire et mesures quasi-invariantes pour un système dynamique. Colloquium Mathematicum, vol. 84/85 part 2 (2000), 457-480.

9. Conze, J.-P. And Raugi, A., On the ergodic decomposition for a cocycle. Colloquium Mathematicum, vol. 117 (2009), 121-156.

10. Davenport, H., On some infinite series involving arithmetical functions, Quart. J. Math., vol 8, pp. 8-13 (1937).

11. Davenport, H., On some infinite series involving arithmetical functions II, Quart. J. Math., vol 8, pp. 313-320 (1937).

12. ERdös, P., On the distribution of convergents for almost all real numbers, J. Number Theory, 2 (1970), 425-441.

13. Feldman, J. And Moore, C., Ergodic equivalence relations, cohomology and von Neumann algebras, I. Trans. Amer. Math. Soc. 234 (1977), no.2, 289-324.

14. Jaffard, S., On Davenport expansions, Fractal geometry and applications : a jubilee of Benoît Mandelbrot. Part 1, 273-303, Proc. Sympos. Pure Math., 72, Part 1, Amer. Math. Soc., Providence, RI, 2004.

15. Khinchin, A., Continued Fractions, The University of Chicago Press, Chicago, IL, 1935.

16. Lemańczyk, M., Analytic non-regular cocycles over irrational rotations, Comment. Math. Univ. Carolin. 36 (1995), no. 4, 727-735.

17. Lemańczyk, M., Parreau, F. And Volný, D., Ergodic properties of real cocycles and pseudo-homogeneous Banach spaces, Trans. of the AMS, vol. 348, no. 12, 1996, 4919-4938.

18. PASK, D., Skew products over the irrational rotation, Israël J. Math. 69 (1990).

19. Pólya, G. And Szegö, G., Problems and theorems in Analysis, vol.1 Springer-Verlag (1978).

20. Schmidt, K., Lectures on Cocycles of Ergodic Transformations Groups, Lecture Notes in Math. vol. 1, Mac Millan Co. of India (1977).

21. Veech, W., Finite group extensions of irrational rotations, Israël Journal of Math., 21, 1975, $240-259$.

22. Zimmer, R., Amenable ergodic group actions and an application to Poisson boundaries of random walks, J. Functional Analysis 27 (1978), no. 3, 350-372.

Laboratoire d'Analyse et de Mathématiques Appliquées, Université Paris-Est, Faculté des Sciences et Technologies, 61 AV. du Général de Gaulle, 94010 Créteil Cedex, FranCE

E-mail address : julien.bremont@u-pec.fr 